\title{
Detection of ventricular fibrillation based on neuro- fuzzy system and phase space reconstruction
}

\author{
Sang-Hong Lee \\ Department of Computer Science \& Engineering, Anyang University, Anyang-si, Republic of Korea
}

\begin{abstract}
This study proposes feature extraction using wavelet transform (WT), sequential increment method, and phase space reconstruction (PSR) to classify normal sinus rhythm (NSR) and ventricular fibrillation (VF) from ECG episodes. We implemented four pre-processing steps to extract features from ECG episodes. In the first step, we use the WT for multi-scale representation and analysis, and then we extract wavelet coefficients from ECG episodes. In the second step, we use sequential increment method to extract peaks from the wavelet coefficients. In the third step, we make a threedimensional phase space reconstruction (PSR) using the successive peaks. In the final step, we calculate the Euclidean distance between the peaks that are plotted in a threedimensional phase space diagram and origin $(0,0)$, and then extract 20 features from the Euclidean distances by using statistical methods, including frequency distributions and their variabilities. We apply the 20 features as inputs to a neural network with weighted fuzzy membership functions (NEWFM), and recorded sensitivity, specificity, and accuracy performances of $100 \%, 100 \%$, and $100 \%$, respectively.
\end{abstract}

Keywords-ventricular fibrillation, automated external defibrillator, neuro-fuzzy system, electrocardiogram, wavelet transform.

\section{Main Idea}

The theory of nonlinear phase space reconstruction (PSR) is proposed by Packard and Takens[1]. It is necessary to develop a statistical technique based on the PSR of NSR and VF for the accurate and timely diagnosis of VF. Phase space reconstruction (PSR) is based on limited data to reconstruct an attractor and study the dynamic behavior of a system. For any time series signal $X_{i}$, where $i=1,2, \ldots, N, N$ is the total number of points. After selecting appropriate embedding dimension $m$ and delay time $\tau$, phase space reconstruction can be immediately conducted. The phase space can be reconstructed according to:

$$
Y_{j}=\left(X_{j}, X_{j+\tau}, X_{j+2 \tau}, \ldots, X_{j+(m-1) \tau}\right)
$$

where $j=1,2, \ldots, N-(m-1) \tau, m$ is the dimension of the phase space, and $\tau$ is the delay time.

In this study, we used successive peaks $X_{i}$ where the $x$ axis plots $X_{i}$, the $y$-axis plots $X_{i+1}$, and the $z$-axis plots $X_{i+2}$. This plot is known as a three-dimensional phase space diagram. Figure 1 shows the successive peaks plotted in a three-dimensional phase space diagram. 3D PSRs of successive peaks in the wavelet coefficients of NSR and VF episode are shown in Figure 1. These figures show that the patterns related to the higher dimensional transformations can be more discriminate than those in the time series itself. Figure 1 also shows that PSR's points of NSR episode accumulate more around the origin than those of the VF episode, and therefore, the NSR episodes are in the phase portraits and are more regular than the VF ones.

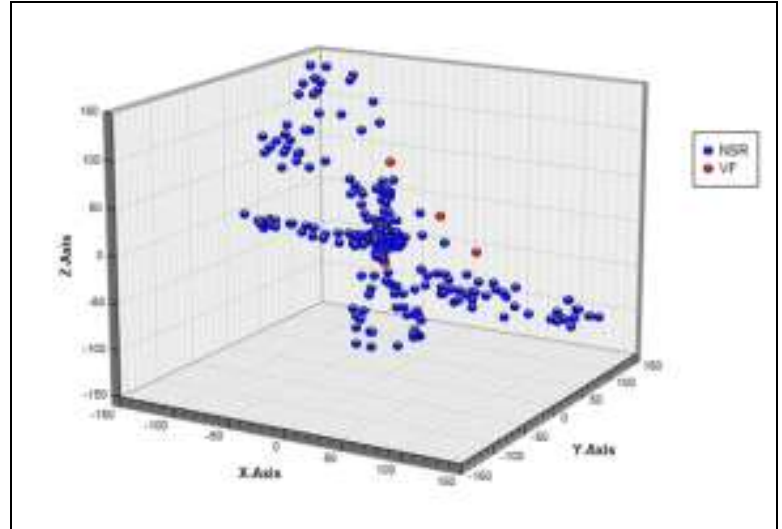

(a) 3D PSR for NSR and VF episode of successive peaks in wavelet detail coefficients D1

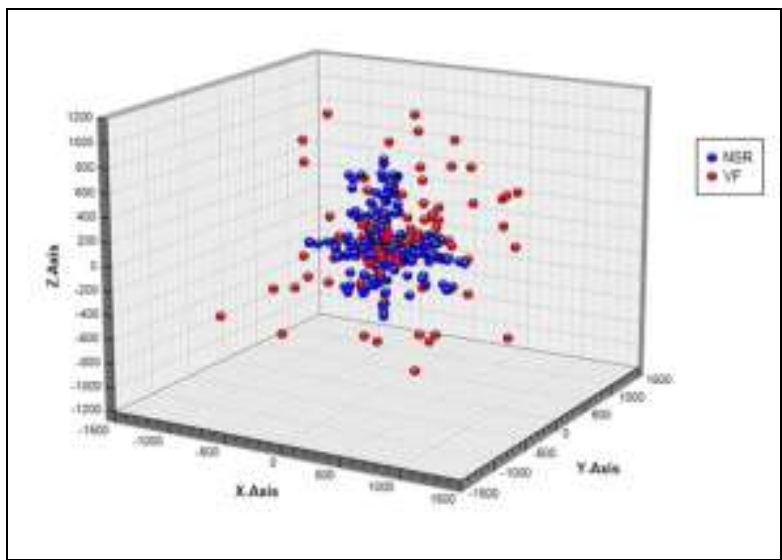

(b) 3D PSR for NSR and VF episode of successive peaks in wavelet detail coefficients D2

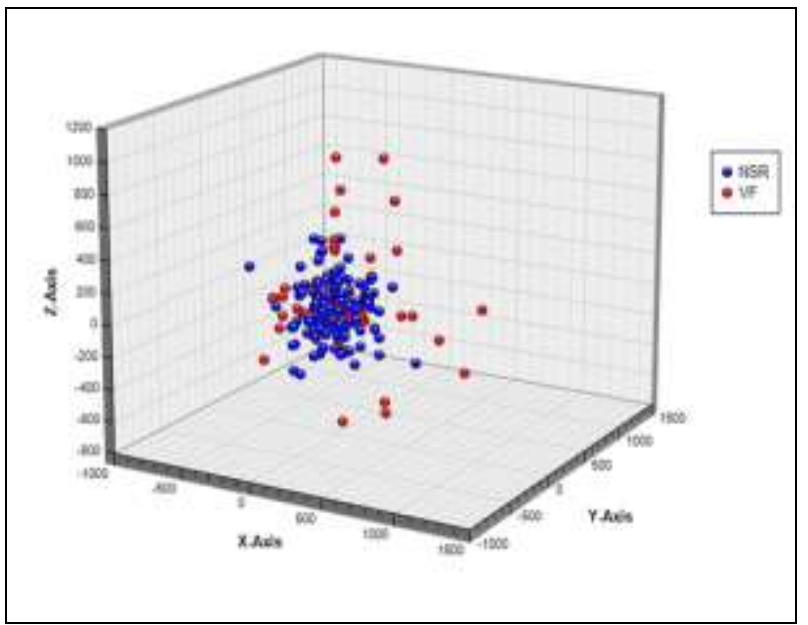

(c) 3D PSR for NSR and VF episode of successive peaks in wavelet detail coefficients D3 


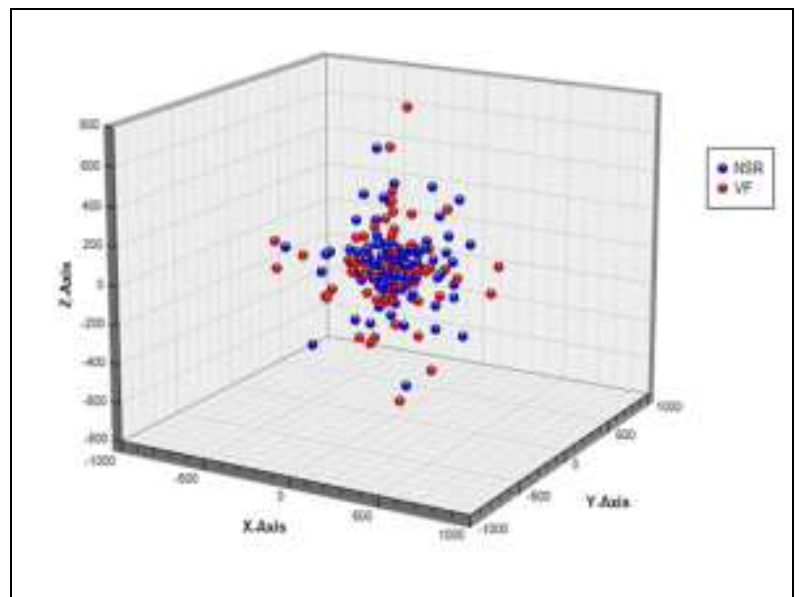

(d) 3D PSR for NSR and VF episode of successive peaks in wavelet detail coefficients D4

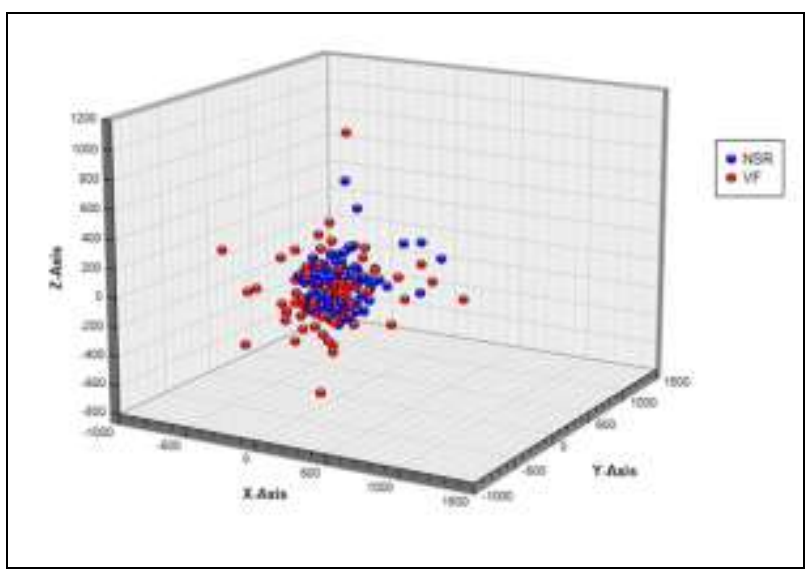

(e) 3D PSR for NSR and VF episode of successive peaks in wavelet approximation coefficients A4

Figure 1. Successive peaks plotted by 3D PSR

\section{Experimental Results}

In this study, we classify NSR and VF episodes from ECG episodes. The classification performance results are shown in TABLE I. TP (true positive) refers to cases where VF episodes are classified as VF episodes, and TN (true negative) refers to cases where NSR episodes are classified as NSR episodes. FN (false negative) refers to cases where VF episodes are classified as NSR episodes, and FP (false positive) refers to cases where NSR episodes are classified as VF episodes. To assess the performance of the NEWFM, it was compared with Amann's PSR[2] and probabilistic neural network[3]. The sensitivity, specificity, and accuracy values presented in TABLE I are computed based on the following equations:

$$
\begin{gathered}
\text { Sensitivity }=\frac{T P}{T P+F N} \times 100 \\
\text { Specificity }=\frac{T N}{T N+F P} \times 100 \\
\text { Accuracy }=\frac{T P+T N}{T P+F N+T N+F P} \times 100
\end{gathered}
$$

TABLE I. COMPARISON OF OTHER APPROACHES AND NEWFM PERFORMANCE RESULTS (\%)

\begin{tabular}{|l|l|c|c|}
\hline & \multicolumn{1}{|c|}{ Accuracy } & Specificity & Sensitivity \\
\hline Amann's PSR [2] & 99.2 & 99.2 & 74.8 \\
\hline PNN [3] & 99.71 & 99.1 & 97.98 \\
\hline NEWFM & 100 & 100 & 100 \\
\hline
\end{tabular}

\section{Acknowledgment}

This research was supported by Basic Science Research Program through the National Research Foundation of Korea (NRF) funded by the Ministry of Education (NRF2014R1A1A2054293).

\section{References}

[1] F. Takens, Detecting strange attractors in turbulence, in: Dynamical Systems and Turbulence, Warwick 1980, Springer, Berlin/Heidelberg (1981) 366-381

[2] A. Amann, R.t Tratnig, K. Unterkofler, Detecting ventricular fibrillation by time-delay methods, IEEE Transactions on Biomedical Engineering 54 (2007) 174-177.

[3] J.-S. Wang, W.-C. Chiang, Y.-L. Hsu, Y.-T. C. Yang, ECG arrhythmia classification using a probabilistic neural network with a feature reduction method, Neurocomputing 116 (2013) 38-45. 